\title{
Three cases with Klippel-Trenaunay syndrome and treatment options
}

\author{
Ersin Çelik ${ }^{1}$, İlker Alat ${ }^{2} \odot$ \\ ${ }^{1}$ Department of Cardiovascular Surgey, Isparta City Hospital, Isparta, Turkey \\ ${ }^{2}$ Department of Cardiovascular Surgey, Afyonkarahisar State Hospital, Afyonkarahisar, Turkey
}

\begin{abstract}
Klippel-Trenaunay syndrome (KTS) is a rare congenital anomaly characterized by capillary malformations, soft tissue and bone hypertrophy and varicosities. The presence of at least two of the three clinical conditions is sufficient for the diagnosis of KTS. Early diagnosis and treatment are important because of possible serious complications such as deep vein thrombosis and pulmonary embolism. Venous system aplasia and/or hypoplasia might be observed in KTS. Therefore, deep venous system must be evaluated definitely before determining the treatment strategy. It's herein presented our conservative and surgical treatment applications in three KTS patients.
\end{abstract}

Keywords: Klippel-Trenaunay syndrome, embryonic, varicose, venous, venous insufficiency, stripping

K lippel-Trenaunay Syndrome (KTS) is a rare congenital anomaly characterized by capillary malformations, soft tissue and bone hypertrophy and varicosities [1]. It was first described by Klippel and Trenaunay in 1900 [2]. The presence of at least two of the three clinical conditions is sufficient for the diagnosis of KTS [3]. A form with soft tissue hypotrophy without bone pathology is defined as "atypical Klippel Trenaunay Syndrome" [4, 5]. Early diagnosis and treatment are important because of possible serious complications such as deep vein thrombosis and pulmonary embolism.

Patients with KTS may usually be treated with conservative methods. However, surgical treatment can be performed in patients with symptomatic varicose veins due to KTS, if they do not have any problem like thrombosis with their deep venous systems [6]. It's herein presented our conservative and surgical treatment applications in three KTS patients.

\section{CASE SERIES PRESENTATION}

\section{Case \# 1}

A 26-year-old male patient was admitted to our outpatient clinic with pain, redness, and hardness in his varicose veins. He stated that he has had varicose veins since his childhood period, and that his leg thickens progressively but that the redness and stiffness has just begun. During his physical examination, it was observed that there were a large number of varicose veins especially in the lateral crural region. Erythematous and sensitive thrombotic venous segments were observed around the ankle. Reddish-pinky hyperpigmented skin lesions on the left lower extremity, extending to the back of the foot were observed (Fig. 1). When compared to the right lower extremity, the left lower extremity was hypertrophic. Colored Doppler ultrasonography (CDU) revealed multiple varicose veins under the skin, acute thrombus 


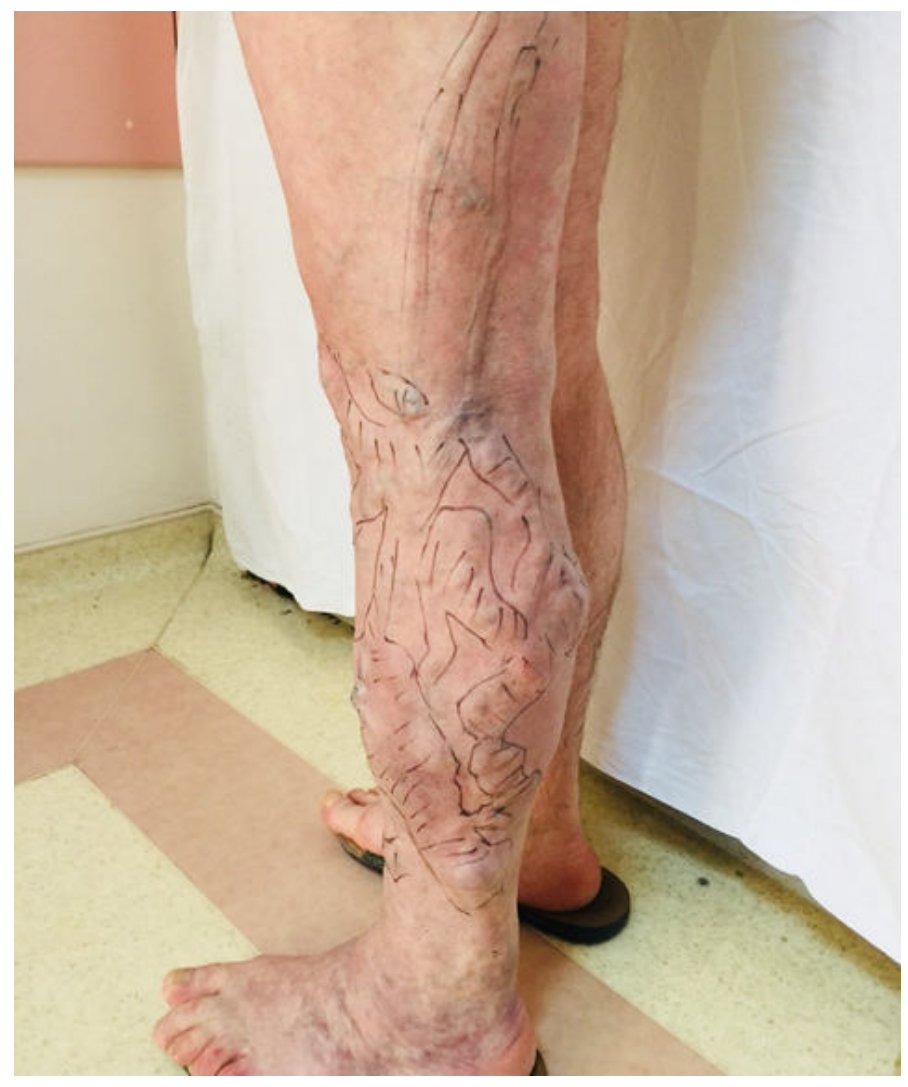

Fig. 1. Reddish-pinky hyperpigmented skin lesions on the left lower extremity, extending to the back of the foot. There are a large number of varicose veins especially in the lateral crural region, and erythematous and sensitive thrombotic venous segments around the ankle.

formation within the varicose veins around the ankle and lateral embryonic varicose vein (LEV). According to CDU evaluations LEV which started at the level of the ankle and joined popliteal vein had severe venous reflux. Deep venous system and the great saphenous vein (GSV) were patent and no reflux was detected. The patient was then given medical therapy for acute thrombophlebitis. Under spinal anesthesia on the 15th day after the treatment, a stripping operation was applied to the LEV below the popliteal entrance. Segmental phlebectomies were performed for the enlarged satellite venous segments. He was discharged on the 1st postoperative day with a compression sock and symptomatic treatment. During the first month follow-up, the patient stated that the pain in his lower extremity decreased significantly and the quality of his life increased.

\section{Case \# 2}

A 24-year-old female with a 24-week pregnancy was referred to our outpatient clnic from the obstetrics and gynecology clinic. She stated that she applied many times to different hospitals since her childhood period due to a growth failure in both her left arm and left leg, birthmarks and vascular enlargement on the skin but that she could not be diagnosed. Physical examination revealed hypoplasia in the soft tissue of her upper and lower left extremities, pervasive reddish-pinky hyperpigmented skin lesions in her upper and lower left extremities, and subcutaneous varicosities in her left lower extremity. X-ray examination for bone structures could not be performed due to pregnancy, but there was no difference in length between the extremities and their functions were normal. No Valsalva maneuver was ordered to undergo due to pregnancy in CDU evaluation. Deep venous system was observed as being patent. No thrombus was observed in deep or superficial venous systems. As a result, the patient was diagnosed with "atypical KTS" with soft tissue hypotrophy. Compression sock was recommended to prevent venous complications and polyclinic control was suggested after delivery.

\section{Case \# 3}

A 19-year-old male patient was admitted to our outpatient clinic with the complaint of increased and distinctive subcutaneous varicose veins in the left lower extremity. According to his medical history, he had been diagnosed with Klippel-Trenaunay Syndrome 3 years ago and had undergone a stripping operation of LEV in his left lower extremity together with segmental phlebectomies by a different cardiovascular surgeon. He hasn't used compression socks for almost a year.

On his physical examination, there were pervasive reddish-pinky hyperpigmented skin lesions and also pervasive subcutaneous varicose enlargements especially in the posterolateral region of his left lower extremity. When compared to the right one, the left lower extremity was hypertrophic. His deep and superficial venous systems were observed as being patent in the CDU examination. Additionally, pervasive varicose enlargements were detected in his subcutaneous tissue. No new surgical intervention was planned. Regular polyclinic controls with compression socks and symptomatic treatments were recommended. 


\section{DISCUSSION}

KTS is composed of soft tissue and bone hypertrophy, capillary hemangiomas and superficial varicose veins often located laterally [7]. Skin lesions and edema are common. Depending on the symptoms and skin attitudes, patients may consult different clinics during their whole life-course. This process, which starts with pediatric admissions in childhood period, might be followed by many different clinical admissions such as dermatology, orthopedics, plastic surgery, cardiovascular surgery. For these reasons, a multidisciplinary approach is required for the diagnosis, follow-up and treatment of the disease according to the patient's symptoms, organ involvement and his/her expectations. KTS is unilateral in $85 \%$ of patients, and $10 \%$ of patients have both upper and lower extremity involvement, as in our second case [8]. LEV is observed in $68-80 \%$ of cases. It drains into iliac vein (5\%), popliteal vein $(11 \%)$, great saphenous vein $(14 \%)$, superficial femoral vein $(17 \%)$, deep femoral vein $(20 \%)$ or gluteal veins (33\%) [9]. Before the operation, LEV and deep venous system have to be evaluated in detail. Venous system aplasia and hypoplasia might be observed in KTS. In a study by Redondo et al., hypoplasia and aplasia in the deep venous system were detected in $22 \%$ of the patients with KTS. In this group of patients, LEV functions as a functional collateral and for that reason surgical intervention is strictly contraindicated in these patients particularly [10]. Conventional venography, magnetic resonance venography, CDU can be used for the examination of the venous system. Although it was stated that venography has an important place in the literature in patients with KTS, it is seen that CDU alone is sufficient to diagnose these cases in many different reports as seen in the report of Usta et al. [11]. Because in the classical definition of KTS, a triad is mentioned in which the presence of two is sufficient. This triad is a rare congenital anomaly characterized by capillary malformations, soft tissue and bone hypertrophy and varicosities [1]. Therefore, if the presence of varicose structures typical for CTS is evident by inspection and you have the opportunity to work with a radiologist whose experience you believe, CDU examination alone may be sufficient to determine the indication for the operation. Moreover, it will also protect the patient from the invasive intervention of venography and from the nephrotoxic drug exposure. For these reasons, due to the presence of our radiologists, whose experience we are sure of, we did not require venography during the evaluation of our cases and decision-making process, and CDU evaluations were found sufficient. We believe that such an algorithm is important not only for the patient but also for cost analysis. It is evident that CDU examination alone in the presence of a wellexperienced radiologist whom the vascular surgeon is sure of him/her will be much more cost effective than venography.

Treatment of CTS is conservative and symptomatic. Elastic compression stockings and venotonic agents may be used for vascular involvement of the lower extremities. Thrombophlebitic conditions that may occur as in our first case should be treated conservatively. Operation should be avoided in the period when thrombophlebitis is active. Surgical intervention can be performed in selected patients whose symptoms persist despite of conservative treatment and whose quality of life decreases $[5,12]$. In our first case, LEV was present and surgical treatment was performed because of the existence of patent deep venous system and venous hypertension symptoms. In our second patient, while conservative treatment was planned because of her pregnancy, third patient was not planned to undergo a surgery, since he was previously operated. He, too was taken with medical treatment only.

Another point to be considered in the differential diagnosis is that KTS should be differentiated from Parkes Weber Syndrome characterized by capillary malformation, hypertrophic extremity, heart failure associated with hyperdynamic arteriovenous fistulas. It was stated that arteriovenous malformation presents the criterion for distinguishing Parkes Weber syndrome from Klippel-Trenaunay syndrome [13].

\section{CONCLUSION}

In conclusion, even though KTS is a rare syndrome, it should be evaluated more carefully than classical venous insufficiency patients. The composition of soft tissue and bone hypertrophies, which are a part of its triad, should be well studied. In 
particular, the condition of the deep venous system should be carefully examined. Because the current status of the deep venous system is one of the most important factors that determine the surgical indication. Most patients with KTS are treated conservatively with elastic compression socks and venoactive agents. However, surgical treatment can be performed if there is a complaint related to venous hypertension-induced pain and venous dilatation in the presence of LEV together with a patent deep venous system. CDU examination alone performed in safe hands may be sufficient to determine the indication for the surgical operation.

\section{Informed consent}

Written informed consent was obtained from the patient for publication of these cases and any accompanying images.

\section{Conflict of interest}

The authors declared that there are no potential conflicts of interest with respect to the research, authorship, and/or publication of this article.

\section{REFERENCES}

1. Oduber CE, van der Horst CM, Chantal MAM, Hennekam RC. Klippel-Trenaunay syndrome: diagnostic criteria and hypothesis on etiology. Ann Plast Surg 2008;60:217-23.

2. Spencer L, Quarmby JW. Klippel-Trenaunay
Syndrome and left iliac vein agenesis. EJVES Extra 2007;13:50-1.

3. Delis KT, Gioviczki P, Wennberg PW, Rooke TW, Driscoll DJ. Hemodynamic impairment, venous segmental disease, and clinical severity scoring in limbs with Klippel-Trenaunay syndrome. J Vasc Surg 2007;45:561-7.

4. Bircher AJ, Koo JY, Frieden IJ, Berger TG. Angiodysplastic syndrome with capillary and venous malformation associated with soft tissue hypotrophy. Dematology 1994;189:292-8.

5. Ada F, Aydın A, Emren SV, Çelik E. A rare lateral embriyonic varicose vein surgery in Klippel-Trenaunay Syndrome with brief review of the literature. Int J Contemp Med Res 2016;3:710-1.

6. Garzon M, Huang JT, Enjolras O, Frieden IJ. Vascular malformations. J Am Acad Dermatol 2007;56:541-7.

7. Zea MI, Hanif M, Habib M, Ansari A. Klippel-Trenaunay sydrome: a case report with brief review of literatüre. J Dermatol Case Rep 2009;3:56-9.

8. Gloviczki P, Holler LH, Telander RL, Kaufman B, Bianco AJ, Stickler GB. . Surgical implication of Klippel-Trenaunay syndrome. Ann Surg 1983;197:353-62.

9. Stephen E, Kota AA, Agarwal S, Selvaraj D, Premkumar P, Ponraj S, et al. Lateral marginal vein: have we understood its significance? Indian J Vasc Endovasc Surg 2017;4:43-5.

10. Redondo P, Bastarrika G, Aguado L, Martinez-Cuesta A, Sierra A, Cabrera J, et al. Foot or hand malformations related to deep venous system anomalies of the lower limb in KlippelTrenaunay syndrome. J Am Acad Dermatol 2009;61:621-8.

11. Usta S, Guday M. Klippel-Trenaunay syndrome. Turk Gogus Kalp Dama 2013;21:179-82.

12. Premkumar P, Stephen E, John JM, Kota AA, Samuel V, Selvaraj D, et al. Managment of Klippel-Trenaunay syndrome from a single center in India: experience shared. Indian J Vasc Endovasc Surg 2018;5:149-53.

13. Banzic I, Brankovic M, Maksimović Ž, Davidović L, Marković M, Rančić Z. Parkes Weber syndrome-Diagnostic and management paradigms: a systematic review. Phlebology 2017;32:371-83. 